\title{
GENERALIZED COEFFICIENTS OF GOODS QUALITY AND SATISFYING OF CONSUMERS DEMAND
}

\author{
Lenar Safiullin', Nail Safiullin², Gulnara N. Ismagilova ${ }^{3}$ \\ ${ }^{1}$ doctor of economics sciences, \\ Department of Economics, Kazan State Agricultural University (Russia), lenar_s@mail.ru \\ ${ }^{2}$ doctor of economics, doctor of technical sciences, \\ Department of Economics, Kazan State Agricultural University (Russia), \\ 3 assistant of department economic theory of the Kazan State Agricultural University
}

\begin{abstract}
Annotation. In process of work the problems are set and solved. There are problems of social welfare research in conditions of nonhomogeneity of objects and subjects of management, of revealing and substantiating insuperable difficulties in rising of social welfare by approaches, worked out before. Also there developed a new direction in implementation of the most important aim of any socially-oriented economy.
\end{abstract}

Key words: consumer demand, quality, qualitative growth of welfare, competitiveness

\section{Introduction}

Increase of goods quality is one of the conditions of actual satisfaction of customers demands (interests). However estimation of goods quality and level of satisfaction is difficult task $[1,2]$.

At present time producers of goods for determining coefficients of quality of objects (goods and services) widely use instrumental and expert methods. Instrumental methods are based on physical effects and use of special apparatuses. Expert methods are used there, where physical phenomenon is not open or very difficult to use. Variety of expert method is so-called organoleptic method, based on use of human organs of sense. There exist some different approaches to estimation of goods quality, which use multi-factor models. The most widespread among them is the method of neighboring point. However known methods lead to different estimations of consequence of consumers relation coefficients.

In spite of existence of different approaches in the sphere of determining of quality coefficients there are some unsolved problems. Difficulties are connected with transformation of the notion of quality from technical category to economical. They began to consider quality in interconnection with demand, satisfaction of consumers demand, and amount of expenses and so on. Quality is an aggregate of features and characteristics of object (goods or service) which enables it to satisfy provided or supposed needs. Consumers demands are characterized both by qualitative and qualitative parameters of goods. In such conditions use of known methods is inconvenient since the abilities of the known methods do not respond to difficulty and many-sided character. In this connection the most difficult and less formalized and actual in theoretical relation and practical use of the notion of quality of objects, oriented on consumers demand, is development of generalized coefficients of quality.

\section{Target setting}

Satisfaction of consumers' demand - is a peculiar measure of concordance of parameters of object to parameters of consumers' demand. Concordance of those parameters can be measured by generalized coefficients of quality, which we suggest to introduce on the basis of choice of corresponding measure. To this measure the following demands are made. Objects and demands of consumers are characterized by arbitrary number of parameters, among which is the definite number of parameters, specified by quantitative data and several parameters only by qualitative data.

Besides even at high enough quality level goods or service can not satisfy consumer (customer), if its fixed price is too high. In other words quality can not be defined without price taken into consideration. It is significant at planning and projecting of quality. Providing of proper quality is impossible without regulation of prices incomes and costs. The same can be said about the volume of supply (production). If enterprise doesn't have data about volume of supply, amount of production waste, rejection rate, or necessary alterations, it is impossible to define the share of faulty units, and failure rate on enterprise. Without those 
coefficients providing of necessary quality level is impossible.

Insufficient volume of supply of goods or service, which is in requisition, causes inconvenience of consumers. Excessive volume of supply means over-expenditure of labor force, raw material, and energy. Regulation of expenses and providing of required level of quality are two sides of one medal. That's why it's always necessary to aim at delivery of goods or services at exactly specified volume, specified level of quality and at defined price. Therefore price and non-price parameters of goods should correspond to consumers' demand. That's why they must be considered by coefficient of quality. Coefficients of quality must allow the successful economic interpretation of obtained results. To our point of view measure of similarity fully corresponds to all these requirements [3]. Hereinafter as a generalized coefficient of quality of object oriented on satisfaction of consumers demand we will use measure of similarity $q_{n k i}=q\left(\Pi_{n i}, T_{k i}\right)$ between $i$-ми parameters $k$-Го goods $T_{k}=\left[T_{k 0}, T k_{l}, \ldots, T_{k i}, \ldots, T_{k I}\right]$ and corresponding $i$-МИ parameters of demand $n$-Го consumer, taken as a base of comparison. $\Pi_{n}=\Pi_{n 0}, \Pi_{n 1}, \ldots, \Pi_{n i}, \ldots, \Pi_{n I}$.

Measure of similarity, being generalized coefficient of quality of goods, connected with measure of closeness of vector parameters $T_{k}$ и $\Pi_{n}$, by the simple ratio $q_{n k}=1-d_{n k}$, $n=1, \bar{N}, k=1, \bar{K}$.

By generalized coefficient of quality we mean measure of similarity $q_{n k}$ between parameters of $k$-го object $T_{k}(k=1, \bar{K}$. $)$ and need of $n$-го consumer $\Pi_{n}(n=1, N$,$) . Coefficient of$ quality is - qualitative measure of consumers' satisfaction on goods and service market. $\left(q_{n k}-\right.$ is positive real number describing quality of goods). Quality is one of determining factors of function of utility and competitiveness.

\section{Bases of comparison}

Precision of estimation of quality and decisions significantly depends on choosing bases of comparison. Starting from the set target, bases of comparison can be:

- consumers' needs;

- value of necessary efficiency;

- hypothetic sample;

- group of analogues .

In case when bases of comparison is needs of consumers, there must be carried out the choice of nomenclature and preset of parameters of customers needs values, parameters of evaluated or competing production, which consumer use estimating production on market, and also ponderability of those parameters in their general set.

When as a base of comparison we take value of production coefficient, needed for consumer, and also amount of funds, which consumer is ready to spend on purchase and consumption, we choose as a standard coefficient itself or the amount of funds.

If estimated product has competitor then product sample models need and acts as materialized demands to which estimated product must correspond.
Sometimes hypothetical sample acts as bases of comparison. It is average value of parameters of a group of items. Such procedure is used in such a case when information on specific sample-analogue is insufficient. In fact we talk about analyses of need which may not exist and this estimation must be considered as preliminary and a subject for further specification.

More often as bases of comparison there is taken group of analogues, singled out according to correspondence of classification parameters of sample and estimated production from which are chosen the most stately and then progressive items, having the best prospective for the further widening of sales volume.

The estimation of satisfaction of production consumers' demands is made by comparison of parameters of analyzed production with parameters of bases of comparison of consumers needs. Comparison is made by groups of technical and economical parameters.

\section{Generalized coefficients of goods quality and consumers satisfaction}

Generalized coefficient of quality and satisfaction of consumers, introduced on the basis of measure of similarity between parameters of object and bases of comparison must correspond to the three following conditions [5]:

1. $q_{n k}=\max q_{n k}$, if $\Pi_{n}=T_{k}$;

2. $q_{n k}=q_{k n}$, if $q\left[\Pi_{n}, T_{k}\right]=q\left[T_{k}, \Pi_{n}\right]$;

3. $0 \leq q_{n k}<\max q_{n k}$, if $\Pi_{n} \neq T_{k}, \Pi_{n}, T_{k} \in \Pi, n=\overline{1, N}, k=\overline{1, K}$,

where $\Pi$ - range of variation of parameters of bases of comparison, for example, consumers needs.

The first condition of quality coefficient is the condition of maximum object correspondence to bases of comparison; for example, satisfaction of consumers means perfect or potentially reachable level of quality.

The second condition is a the condition of symmetry, and the third condition satisfies the request of monotone decrease of quality coefficient (measure of similarity) $q_{n k}$ on distance $d_{n k}$ i.e. из $d_{n k} \geq d_{n j}$ with the necessity there follows satisfaction of inequality $q_{n k} \leq q_{n j}$.

The case of equality of maximum value of quality coefficient to unity i.e. $\quad q_{n k}=\frac{q_{n k}}{q\left(\Pi_{n}, \Pi_{n}\right)}=1 \quad$ corresponds to normalized value of generalized coefficient of quality and is introduced as follows:

1. $q_{n k}=1$, if $\Pi_{n}=T_{k} ; q_{n k}=\frac{q_{n k}}{q\left(\Pi_{n}, \Pi_{n}\right)}$;

2. $q_{n k}=q_{k n}$, if $q\left[\Pi_{n}, T_{k}\right]=q\left[T_{k}, \Pi_{n}\right]$;

3. $0 \leq q_{n k} \leq 1$, if $\Pi_{n} \neq T_{k}$ for all $\Pi_{n}, T_{k} \in \Pi, n=\overline{1, N}, k=\overline{1, K}$.

Function of measure of similarity (2) has homogeneity property towards arguments $\Pi_{n i}$ и $T_{k i}$, because following condition is fulfilled for it $q\left(\lambda_{i} \Pi_{n i}, \lambda_{i} T_{k i}\right)=\lambda_{i}^{r} q\left(\Pi_{n i}, T_{k i}\right)$ where $\lambda_{i}$ и $r$ some numbers. Supposing $r=1, \lambda_{i}=\frac{1}{\Pi_{n i}}$ or 
$\lambda_{i}=\frac{1}{T_{k i}}$. Then the following relations are correct $q\left(\Pi_{n i}, T_{k i}\right)=q\left(1, \frac{T_{k i}}{\Pi_{n i}}\right)=q\left(\frac{\Pi_{n i}}{T_{k i}}, 1\right)$, which are the result of function's homogeneity.

Economic meaning of symmetry of measure of similarity is connected with its single-valued possibility of application of the given coefficient, both by consumers and producers.

In the area of research of consumers' relation the measure of similarity can be used as coefficient of consumers and goods relations. Coefficient of consumers to object relations - is value of measure of similarity between parameters of consumers needs and goods. During research of goods quality on market the measure of similarity is used as coefficient of goods quality.

It should be noted that analogues of quality coefficient are ordinary weigh-scales or measuring bar "meter". They are used both by sellers and customers for determining of weigh or measuring of lengths of goods.

Therefore, coefficient of quality - is the value of the measure of similarity between parameters of comparison bases and object (goods or service).

\section{Analyses and evaluation of goods quality and satisfaction of consumers wants}

Comparing among themselves quality coefficients of objects in relation to each consumer, it's able to determine level of quality of each goods in relation to other goods.

Obviously quality of $j$-2o goods in relation to $n$-st consumer will be determined by formula:

$$
q_{n j}=\max \left\{q_{n 1}, q_{n 2}, \ldots, q_{n j}, \ldots, q_{n K}\right\}, n=\overline{1, N} .
$$

It's also able to introduce relation of $l$-st consumer to the level of quality of $k$-st goods:

$$
q_{l k}=\max \left\{q_{1 k}, q_{2 k}, \ldots, q_{l k}, \ldots, q_{N k}\right\}, k=\overline{1, K} .
$$

Naturally it's necessary to compare value with some threshold value of quality $h_{j}$, which is determined in each case in its own way. Exceeding the threshold level by the coefficient of quality is written as

$$
1-q_{n j}>h_{j}, n=\overline{1, N}, j=\overline{1, J},
$$

and $1>n_{j}$ is the condition of sufficing on market $j$-2o goods to $n$ - y consumer.

Besides let's introduce difference threshold of goods by quality:

$$
q_{n j}-q_{n k} \mid \leq \varepsilon_{j, k}, k \neq j
$$

where - minimum difference between coefficients of goods quality. By the threshold of difference we mean the smallest change of quality coefficient between goods, which consumer is able to notice. Otherwise $n$ - $\breve{u}$ consumer with the same possibility can be satisfied by $j-\mu$ goods or $k-\mu$ goods.

\section{Certain forms of generalized coefficient of quality}

Let's consider certain forms of generalized coefficient of quality as applied to parameters of consumers' wants and goods, quantitatively and qualitatively defined.

To quantitative parameters belong price coefficients and characteristics of goods, defined quantitatively. Coefficients of quality for such parameters can be formed on the basis of use of particular kinds of measure of similarity, metrics of nearness (distance) $d_{n k}$, connected to the measure of similarity $q_{n k}=1-d_{n k}$ and parametrical correlation methods.

Suppose that on finite aggregate of positive parameters of consumers wants $\Pi_{n}$ and goods $T_{k}$ it is required to determine the measure of similarity. Suppose that $\Pi_{n 1}, \Pi_{n 2}, \ldots$, $\Pi_{n i}, \ldots, \Pi_{n I}$ и $T_{k 1}, T_{k 2}, \ldots, T_{k i}, \ldots, T_{k I}$ any positive numbers. Then the expression of unit coefficient of quality is determined as:

$$
q_{n k i}=\frac{2 \sqrt{\Pi_{n i} \cdot T_{k i}}}{\Pi_{n i}+T_{k i}}=\frac{2 \sqrt[T_{k i}]{\Pi_{n i}}}{1+T_{k i} / \Pi_{n i}}=\frac{2 \Pi_{n i} / T_{k i}}{1+\Pi_{n i} / T_{k i}}, i=\overline{1, I},
$$

complies with all three conditions of the measure of similarity

1. $q_{n k i}=\frac{2 \sqrt{\Pi_{n i} \cdot T_{k i}}}{\Pi_{n i}+T_{k i}}=\max q_{n k i}$, если $\Pi_{n i}=T_{k i}$,

2. $q_{n k i}=\frac{2 \sqrt{\Pi_{n i} \cdot T_{k i}}}{\Pi_{n i}+T_{k i}}=\frac{2 \sqrt{T_{k i} \cdot \Pi_{n i}}}{T_{k i}+\Pi_{n i}}=q_{k n i}$ symmetry condition of measure of similarity

3. $0 \leq \frac{2 \sqrt{\Pi_{n i} \cdot T_{k i}}}{\Pi_{n i}+T_{k i}} \leq \max q_{n k i}$, if $\Pi_{n i} \neq T_{k i}$ for all $\Pi_{n i}, T_{k i} \in \Pi_{i}, n=\overline{1, N}$, $k=\overline{1, K}, i=\overline{1, I}$.

For the proof let's consider difference. $\Pi_{n i}+T_{k i}-2 \sqrt{\Pi_{n i} \cdot T_{k i}} \geq 0$, which comes from the third expression (8) Then the correlation $\left(\sqrt{\Pi_{n i}}-\sqrt{T_{k i}}\right)^{2} \geq 0$ is just. Equal sign $\sqrt{\Pi_{n i}}-\sqrt{T_{k i}}=0$ takes place then and only then, when $\Pi_{n i}=T_{k i}$.

Genuinely inserting (8) into the first expression we shall obtain $q_{n k i}=\max q_{n k i}$, if $\Pi_{n i}=T_{k i}$.

This case corresponds to ideal or potential quality of goods by $i$-my qualitative parameter.

Let's consider another condition $0 \leq q_{n k i}=\frac{2 \sqrt{\Pi_{n i} \cdot T_{k i}}}{\Pi_{n i}+T_{k i}}$.

From this condition succeeds that $\Pi_{n i} \neq 0 \quad 0 \leq \frac{2 \sqrt{\Pi_{n i} \cdot T_{k i}}}{\Pi_{n i}+T_{k i}}<2 \sqrt{\Pi_{n i} \cdot T_{k i}}=0$ then and only then, when $T_{k i}=0$. 
This condition means that $\mathrm{i}-\breve{\mathrm{n}}$ parameter of goods $\mathrm{T}_{\mathrm{k}}$ completely do not satisfy consumers demand. As it seen from the expression (9), condition of symmetry is also fulfilled. Thus the expression (8) is the measure of similarity.

If parameters of consumer's demand and goods consist of positive and negative numbers, then the measure of similarity (8) can be given in a little changed way:

$$
q_{n k i}=\frac{2 \Pi_{n i} \cdot T_{k i}}{\Pi_{n i}{ }^{2}+T_{k i}{ }^{2}}
$$

For life-cycle phase, parameters of consumers wants $\Pi_{n i}(t)$ and object $T_{k i}(t)$ are continuous functions of time $t$. Then the measure of similarity (7) and (9) respectively, will be written in integral form..

It should be also noted that as a measure of similarity can be used parametrical and non-parametrical methods of estimation of correlation connections between vectors $T_{k}$ and $\Pi_{n}$, widely used in different statistic and economic researches.

Correlation, translated from English literally means correspondence or accordance. In this context nonparametrical methods of correlation connections must be considered in context of correspondence of satisfaction of qualitatively given parameters of goods and consumers wants. Then as a measure of similarity there are used coefficients of correlations between parameters of consumers' wants and goods. Among these are coefficients of correlation of Spearman, Candall, coefficients of concordance, association, contingency, and others [4]. These coefficients for direct relationship of parameters of demand and goods are measured in limits $q_{n k} \in[0,1]$ and for inverse relationship respectively $-q_{n k} \in[0,1]$.

Generalized (integral) coefficient of quality of price and non-price parameters of goods is evaluated by expression

$q_{n k}=\frac{1}{2}\left[1-\left|\frac{\Pi_{n 0}-T_{k 0}}{\Pi_{n 0}+T_{k 0} \mid}\right|+\frac{1}{I} \sum_{i=1}^{I} q_{n k i}+\frac{1}{J} \sum_{j=1}^{J} q_{n k j}\right]$,

Where $I$ и $J$ - Respectively number of quantitative and qualitative parameters of consumer's demand and object.

Let's consider generalized coefficient of quality $q_{n k}$ on the basis of use of distance $d_{n k i}$ between parameters $\Pi_{n i}, i=$ $\overline{1, l}$ of wants $N$ of consumer and parameters $T_{k i}, i=\overline{1, l}$ of goods of one type $K$

$\left.q_{n k}=\frac{1}{2}\left[1-\left|\frac{\Pi_{n 0}-T_{k 0}}{\Pi_{n 0}+T_{k 0}}\right|+\frac{1}{I} \sum_{i=1}^{I}\left(1-d_{n k i}\right)\right], n=\overline{1, N}, k=\overline{1, K} .1\right)$

where parameters $T_{k 0}$ и $\Pi_{n 0}$ - relatively are price of $\kappa$-гo goods and consumer value of this goods for $n$-2o consumer.
Price of $\kappa$-2o goods $T_{k 0}$ forms from prime cost of goods and profit of producer. Consumer value of goods $\Pi_{n 0}-$ is maximum price, which consumer considers advantageous to pay for it. It consists of consumer cost, equal to price of goods, and unpaid part of consumer value, which is equal to additional profit gained by consumer from use of goods.

The first multiplier in (11) is equal to relation of goods to consumer value and contains in its compound costs and income, both of producer and consumer.

That's why this multiplier determines economic share in coefficient of quality. The second multiplier (radicand) in formula (11) determines technical aspect of quality.

Thus the value $q_{n k}$ simultaneously accounts for correlation with bases of comparison and economical and technical properties of goods.

Metrics of nearness - Euclidian and hemming distances and their modifications are used comparatively wide.[2]

\section{Conclusions}

This work offers methodology and methods of evaluation of quality of objects and level of consumer demands satisfaction. On the basis of use of measure of similarity there were developed generalized coefficients of quality. There were introduced specific types of generalized coefficients of quality. There was substantiated the choice of bases of comparison resulted from task setting. The results can be applied in the sphere of quality management and organizing of production in different branches.

\section{References}

1. Isikava K. Japanese method of quality - M.: Economy, 1998. $215 \mathrm{p}$.

2. Safiullin N.Z., Safiullin L.N Competitive of advantage and competitiveness (monograph.). - Kazan: Publishing house KGU, 2002. - 120 p.

3. Safiullin N.Z.. Stochastic system. Kazan, 1998, 168 p.

4. Gambu M. Klaster analisys .-M.: Finace. 1988. - 342 p.

5. Safiullin N.Z. Multivariate the market: the theory and methodology (monograph.). - Kazan: Publishing house KGU, 2002. -214 p.

6. Safiullin N.Z., Faizrahmanov M.D. Development of business in innovative economy (monograph.). - Kazan: Publishing house KGU, 2007. - 160 p. 